\title{
Predicting the origins of anti-blood group antibody specificity: a case study of the ABO A- and B-antigens
}

\author{
Spandana Makeneni ${ }^{1}$, Ye Ji ${ }^{1}$, David C. Watson ${ }^{2}$, N. Martin Young ${ }^{2}$ and Robert J. Woods ${ }^{1,3}$ * \\ 1 Complex Carbohydrate Research Center, University of Georgia, Athens, GA, USA \\ ${ }^{2}$ Human Health Therapeutics, National Research Council Canada, Ottawa, ON, Canada \\ ${ }^{3}$ School of Chemistry, National University of Ireland, Galway, Ireland
}

Edited by:

Elizabeth Yuriev, Monash University, Australia

\section{Reviewed by:}

Jessica Kate Holien, St. Vincents Institute of Medical Research, Australia

Ricardo Mancera, Curtin University, Australia

*Correspondence:

Robert J. Woods, Complex

Carbohydrate Research Center, University of Georgia, 315 Riverbend

Road, Athens, GA 30602, USA

e-mail:rwoods@ccrc.uga.edu
The ABO blood group system is the most important blood type system in human transfusion medicine. Here, we explore the specificity of antibody recognition toward ABO blood group antigens using computational modeling and biolayer interferometry. Automated docking and molecular dynamics simulations were used to explore the origin of the specificity of an anti-blood group A antibody variable fragment ( $F \vee A C 1001)$. The analysis predicts a number of $\mathrm{Fv}$-antigen interactions that contribute to affinity, including a hydrogen bond between a His ${ }^{\llcorner 49}$ and the carbonyl moiety of the GalNAc in antigen A. This interaction was consistent with the dependence of affinity on $\mathrm{pH}$, as measured experimentally; at lower $\mathrm{pH}$ there is an increase in binding affinity. Binding energy calculations provide unique insight into the origin of interaction energies at a per-residue level in both the scFv and the trisaccharide antigen. The calculations indicate that while the antibody can accommodate both blood group $A$ and $B$ antigens in its combining site, the $A$ antigen is preferred by $4 \mathrm{kcal} / \mathrm{mol}$, consistent with the lack of binding observed for the $B$ antigen.

\section{INTRODUCTION}

Since its discovery in 1900 (1), the ABO blood group system has played a crucial role in defining human blood and tissue compatibility. The blood type of an individual indicates the presence or absence of relevant antigens and antibodies. The three blood types share a core oligosaccharide antigen $(\mathrm{H})$, and based on the glycosyl transferases inherited, different antigens are synthesized (2-4); type A transferase adds a terminal non-reducing $\mathrm{N}$-acetylgalactosamine (GalNAc) residue; type B transferase adds galactose (Gal), whereas individuals with blood group $\mathrm{O}$ retain the unmodified $\mathrm{H}$ antigen. During the first years of life, the immune system forms antibodies upon exposure to non-self antigens from various exogenous factors. Thus an A-type individual will have circulating antibodies specific for the B-antigen, and vice-versa. The high degree of specificity is notable given that the only difference between the structures of the A- and B-antigens is the replacement of an acetamido moiety (in A) with a hydroxyl group (in B). Because of the presence of circulating antibodies, a mismatched blood transfusion or organ transplant can lead to hyperacute immune response and death $(5,6)$. Additionally, under certain circumstances, incompatibilities in blood groups between mother and child can trigger the mother's immune system to produce antibodies against the fetus, causing hemolytic disease (7).

Alterations in the structures of the $\mathrm{ABO}$ antigens often occur during carcinogenesis and therefore they have also been considered tumor markers $(8,9)$. Recently, strong correlations have been established between the presence of particular $\mathrm{ABO}$ and Lewis antigens and susceptibility to infectious diseases, such as Helicobacter pylori, norovirus, and cholera (10), wherein the blood group antigens can be exploited as receptors for bacterial and viral adhesion. Conversely, it has been suggested that endogenous anti-blood group antibodies can recognize blood-group-like carbohydrate antigens on pathogen surfaces, conferring protection against infection (11).

Despite their clinical importance, relatively little is known about the structural basis for these highly specific antibodiesantigen interactions. Although X-ray crystallography has been used to characterize antibody-carbohydrate complexes, the generally enhanced flexibility and conformational heterogeneity of oligosaccharides detracts from the ability to generate co-crystals (12). Additionally, anti-carbohydrate antibodies bind to their antigens with an affinity that is $3-5$ orders of magnitude lower than typical antibodies that bind to protein or peptide antigens. Difficulties in generating 3D structures for carbohydrate-antibody complexes have led to the increasing use of theoretical structure prediction methods $(13,14)$, which, while convenient, are prone to predicting false positives due to inaccuracies in pose scoring functions (15) and to the omission of carbohydrate conformational preferences (16).

In this study, we examined the structural origin of the antigenicity (the specificity and affinity) of a monoclonal antibody raised against blood group A (BGA) antigen, for which an apo structure of the single-chain variable fragment ( $\mathrm{scFv} \mathrm{AC1001)}$ has been reported (17). The specificity data from screening two independent glycan arrays [Consortium for Functional Glycomics (v4.0, request ID: 1808) and from the group of Jeff Gildersleeve] confirmed that the scFv displayed no detectable binding to any $\mathrm{B}$-antigens and only bound to BGA-containing glycans. To provide a structural interpretation for the specificity of AC1001 for BGA over blood groups $\mathrm{H}(\mathrm{BGH})$ and $\mathrm{B}(\mathrm{BGB})$, we generated a 
3D model of the immune complex using molecular docking and refined it by molecular dynamics (MD) simulation. Despite its limitations, molecular docking, with or without additional experimental constraints, such as from NMR data, is often the only approach that may be employed to generate the structure of a ligand-protein complex, in the absence of direct crystallographic data. To enhance the success rate, a recent carbohydrate conformational energy function (16) was employed with AutoDock VINA (18), which quantifies the conformational preferences of oligosaccharides based on their glycosidic torsion angles. MD simulations (50 ns) were subsequently performed to ensure that the docked complexes were stable under physically realistic conditions, and in that event, the MD data were employed in binding free energy calculations. A particular advantage of MD-based energy calculations is that they provide statistically converged values that may be partitioned into contributions from individual residues in the protein and ligand (19).

\section{MATERIALS AND METHODS CLONING, EXPRESSION, AND PURIFICATION OF sCFv}

An scFv gene containing a short linker (RADAA) and the Leu $103 \mathrm{H}$ Val mutation (17), with a $\mathrm{His}_{6}$ tag, was assembled by PCR and cloned into the phagemid pSK4. The construct was maintained in Escherichia coli TG1 cells. Cells from positive clones, as judged by DNA sequence analysis, were grown in minimal media, induced, and subjected to periplasmic extraction. The scFv dimer was purified from the extract by $\mathrm{Ni}^{2+}$ immobilized metal affinity chromatography, by elution with an imidazole gradient.

\section{BIOLAYER INTERFEROMETRY}

Affinity measurements were performed on a biolayer interferometer (Octet Red96, ForteBio). Data were processed using the Data Acquisition and Analysis 8.0 software (ForteBio), and kinetic binding constants were determined from a 1:1 binding model using the OriginPro software (OriginLab). The scFv was immobilized on an amine reactive second-generation (AR2G) biosensor (Lot No. 1311212, ForteBio). The BGA trisaccharide was analyzed as the conjugate to bovine serum albumin (BSA-BGA) and was dissolved in an analysis buffer containing $10 \mathrm{mM}$ HEPES, $150 \mathrm{mM}$ $\mathrm{NaCl}, 3.4 \mathrm{mM}$ EDTA, and $0.005 \%$ Tween 20 at a range of $\mathrm{pH}$ values $(5,5.5,6,6.5$, and 7$)$. A BSA-Le ${ }^{\mathrm{X}}$ trisaccharide conjugate (Prod. No. NGP0302, V-Labs, Inc.) and BSA (Prod. No. 23209, Pierce Thermo Scientific, Rockford, IL, USA) were used as negative controls. Details of the biolayer interferometry (BLI) conditions are provided in Supplemental Material.

\section{AUTOMATED DOCKING}

Docking was performed using AutoDock VINA (18) with 20 docked poses generated for each experiment. The protein and the ligand files were prepared using Autodock tools (ADT) (20) with Gassteiger (21) partial atomic charges assigned to both the protein and ligand residues. The crystal structure of the scFv (PDB ID: 1JV5) was employed, together with a 3D structure of BGA obtained from the GLYCAM-Web server (www.glycam.org). Crystal waters were removed prior to docking and hydrogen atoms were added to the protein using ADT, whereas hydrogen atoms in the ligand were assigned from the GLYCAM residue templates. The glycosidic $\phi$ and $\varphi$ torsion angles were allowed to be flexible during docking, as were all the hydroxyl groups. The protein was maintained rigid. The docking grid box (dimensions: $26.25 \AA \times 26.25 \AA \times 37.5 \AA$ ) was centered relative to the complementarity determining regions (CDRs) of the antibody as described previously (16). For the mutational-docking approach, $\operatorname{Trp}^{\mathrm{H} 100}$ was mutated to Ala by deleting the side-chain atoms of the Trp residue in the crystal structure, followed by processing with the tleap module in AMBER (22). Ala ${ }^{\mathrm{H} 100}$ was reverted back to Trp by restoring the crystal coordinates of the side chain of $\operatorname{Trp}^{\mathrm{H} 100}$. The docked poses from the mutational approach were filtered based on the clashes with the reverted Trp. Poses in which the clashes could not be eliminated by implicit energy minimization (details are in the "MD simulations" section) were rejected. Ligand conformations of all the docked poses from both the flexible and mutational-docking approaches were scored using the recently reported carbohydrate intrinsic (CHI) energy scoring function (16). Any conformations with total $\mathrm{CHI}$-energies $>5 \mathrm{kcal} / \mathrm{mol}$ were rejected. The BGB complex was generated directly from that generated for BGA by simple replacement of the NAc group by an $\mathrm{OH}$ group.

\section{SIMULATIONS}

All the MD simulations were performed with the GPU implementation of the pmed code, pmed.cud_SPDP (23), from AMBER12 (22). The calculations employed the ff99SSB (24) parameters for the protein and the GLYCAM06h (25) parameters for the carbohydrate. For the BGA, BGB-scFv complex simulations, an implicit solvent energy minimization (5000 steps of steepest descent followed by 5000 steps of conjugate gradient), were performed to optimize the side-chain positions of the reverted Trp residue. During this minimization, the backbone atoms of the framework regions were restrained with a $5 \mathrm{kcal} / \mathrm{mol} \AA^{2}$ while the CDRs and the ligand were allowed to be flexible. The systems were then solvated in a cubic water box [120 $\AA$ per side, with a TIP3P water (26)]. Each system was energy minimized using explicit solvent (10,000 steps of steepest descent, 10,000 steps of conjugate gradient). During this energy minimization, the protein residues were restrained with a force constant of $100 \mathrm{kcal} / \mathrm{mol} \AA^{2}$ allowing only the solvent and ligand to relax. This minimization was followed by heating from 5 to $300 \mathrm{~K}$ over the course of 50 ps at constant volume. Production MD simulations were performed for $50 \mathrm{~ns}$ at constant pressure (NPT ensemble) with the temperature held constant at $300 \mathrm{~K}$ using a Langevin thermostat. During the heating and the production $\mathrm{MD}$, the backbone atoms of the protein were restrained with a force constant of $5 \mathrm{kcal} / \mathrm{mol} \AA^{2}$, with the protein side chains and ligand atoms allowed to be flexible. The backbone atoms were restrained in order to ensure that the protein fold remained stable during the course of the simulation. For the BGA trisaccharide MD simulation, the system was solvated in a cubic water box ( $120 \AA$ per side, with a TIP3P water) and energy minimized using explicit solvent (5000 steps of steepest descent, 5000 steps of conjugate gradient). This was followed by heating from 5 to $300 \mathrm{~K}$ for a period of 50 ps at constant volume. Production MD simulations were performed for $50 \mathrm{~ns}$ at constant pressure (NPT). During the minimization, heating, and production MD simulations, there were no restraints placed on the trisaccharide. For both BGA, BGB-scFv complexes and BGA 
trisaccharide simulations, all covalent bonds involving hydrogen atoms were constrained using the SHAKE (27) algorithm, allowing a time step of 2 fs. A non-bonded cut-off of $8 \AA$ was used and longrange electrostatics were employed using the particle mesh Ewald (PME) method (28). Snapshots were collected at 1 ps intervals for subsequent analysis.

\section{ANALYSIS}

The stability of the complexes was assessed by monitoring the root-mean-squared-displacement (RMSD) of the ligand position, the glycosidic torsion angles, the ring conformations, and the protein-ligand hydrogen bonds. All these values except for the ring conformation analysis were generated using the ptraj module of AMBERTOOLS 12 (29). Ligand RMSD values were calculated for the ring atoms, relative to the first time step of the simulation. Hydrogen bond interactions between the protein and the ligand were measured with distance and angle cut-off values of $3.5 \AA$ and $120^{\circ}$, respectively. The ring conformations of each individual residue in the ligand during the course of simulation were analyzed using the recently reported BFMP method (Makeneni et al., submitted). Binding free energies were calculated with the MM-GBSA $(30,31)$ module in AMBERTOOLS12. All the water molecules were removed prior to the MM-GBSA calculation, and desolvation free energies were approximated using the generalized born implicit solvation model $($ igb $=2)(32)$.

\section{RESULTS AND DISCUSSION \\ DOCKING ANALYSIS}

In preliminary experiments, docking to the rigid $\mathrm{scFv}$ structure yielded complexes that failed to remain stable during subsequent $10 \mathrm{~ns}$ MD simulations (Table S1 in Supplementary Material). The spontaneous dissociation of the complex during $\mathrm{MD}$ simulation suggested that the docking had failed to detect the correct, high affinity, pose (33). Upon inspection of the MD data, it was observed that light chain residue His49 ( $\mathrm{His}^{\mathrm{L} 49}$ ) forms a stacking interaction with heavy chain residue Trp100 $\left(\operatorname{Tr} \mathrm{p}^{\mathrm{H} 100}\right)$, which occupies a large volume of the presumed binding site, potentially preventing deeper penetration of the ligand (Figure 1).

As Trp residues can also form stacking interactions with the apolar face of monosaccharides in antibody complexes (34), we hypothesized that the trisaccharide ligand might compete for formation of such an interaction with $\operatorname{Tr}^{\mathrm{H} 100}$. For example, the galactose (Gal) residue in a Salmonella trisaccharide antigen stacks against $\operatorname{Trp}^{\mathrm{L} 93}$ in the complex with Fab Se155-4 (34). In addition, in the same complex, $\operatorname{Trp}^{\mathrm{H} 33}$ stacks against the $\mathrm{C}-6$ position in the 6-deoxy sugar Abequose. The BGA antigen contains GalNAc and a 6-deoxy monosaccharide (fucose, Fuc), thus a revised docking experiment was sought that would permit the formation of such interactions with the aromatic residues in the binding pocket. Thus, two alternative docking experiments were designed: in the first, the side-chain torsion angles of $\operatorname{Trp}^{\mathrm{H} 100}$ were allowed to be flexible during docking (termed flexible residue docking); while in the second, Trp ${ }^{\mathrm{H} 100}$ was mutated to Ala prior to docking, and then reverted back to Trp after docking (mutational residue docking). The docked poses were filtered based on three criteria. First, poses in which the GalNAc was not located within the binding pocket were eliminated (Figure 2C). This criterion was adopted based on the results from two array screenings, which indicated that the antibody interacts exclusively with the BGA antigens (Tables S2 and S3 in Supplementary Material) and because the only structural difference between BGA and BGB is the presence of the NAc moiety in the former. Therefore it was hypothesized that the ability of the antibody to discriminate between these two antigens

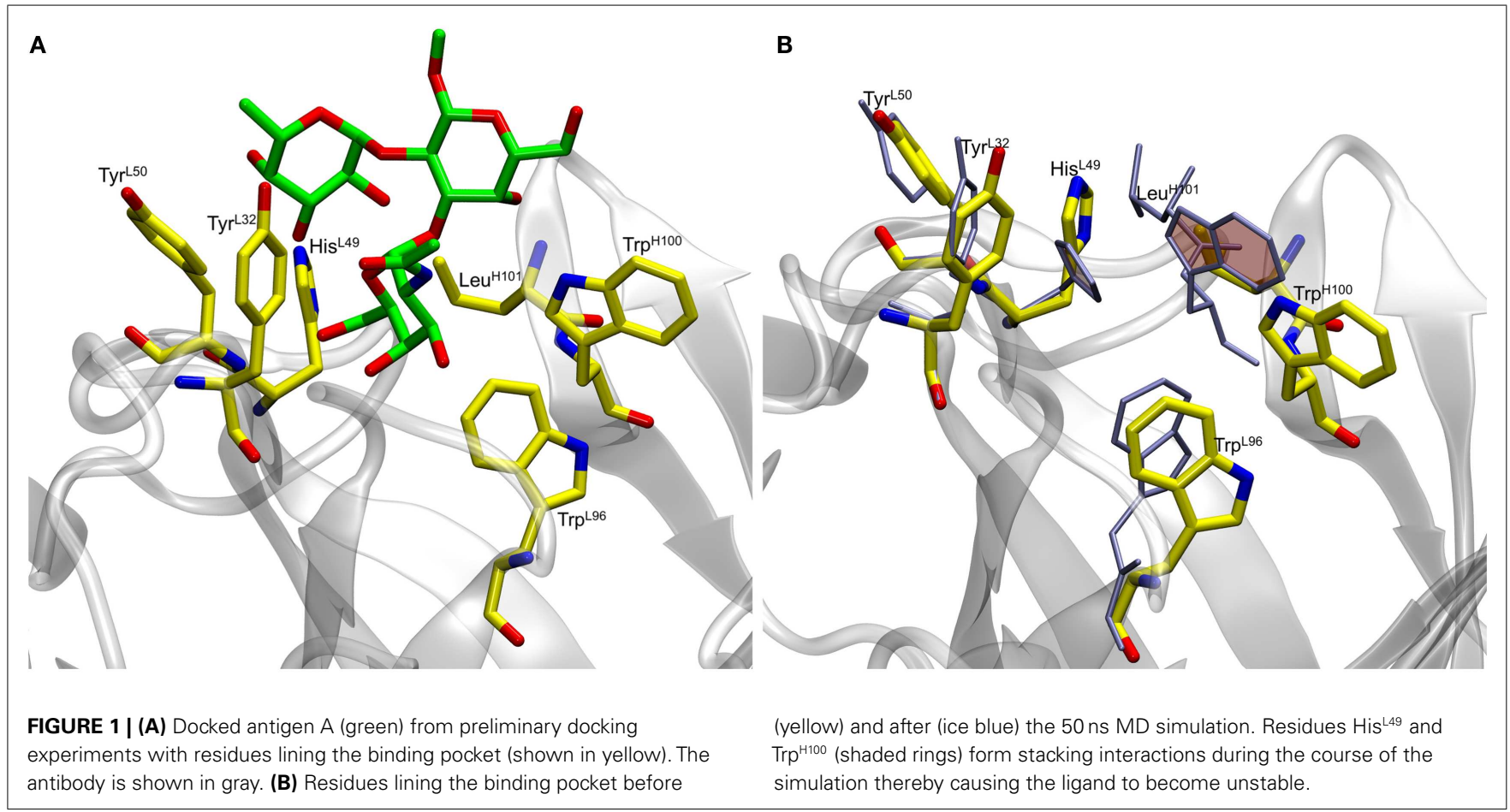



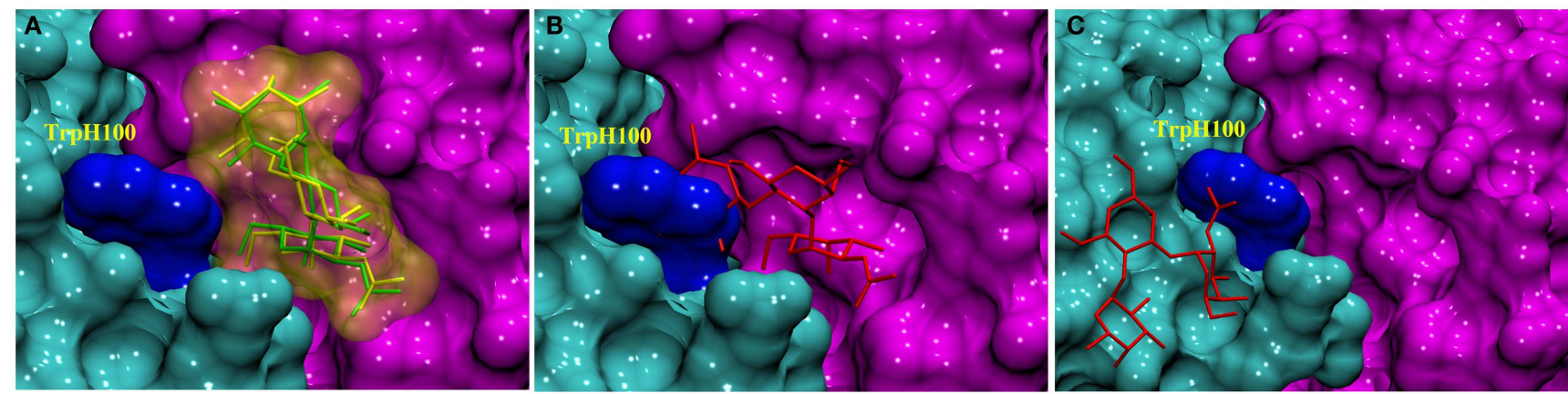

FIGURE 2 | Docked complexes of BGA (stick structure) in the scFv binding site (heavy and light chains shown as solvent accessible surfaces in cyan and pink, respectively, the $\operatorname{Trp}^{\mathrm{H} 100}$ surface is shown in dark blue). (A) The stick structures in green and yellow represent the best-docked poses from the $\operatorname{Trp}^{\mathrm{H} 100}$-mutagenesis and the flexible residue docking approaches, respectively. (B) An example of a docked pose (red) that was eliminated on the basis of clashes ensuing from the Ala ${ }^{\mathrm{H} 100} \mathrm{Trp}$ mutation. (C) An example of a docked pose (red) that was eliminated on the basis of the orientation of the ligand in the binding pocket.

Table 1 | Comparison of glycosidic torsion angles between experimentally observed values and average values obtained from the MD simulations.

$$
\left(\phi_{1}, \varphi_{1}\right)^{\mathbf{a}}
$$

$\left(\phi_{2}, \varphi_{2}\right)^{\mathrm{b}}$

\begin{tabular}{lllll}
\cline { 3 - 4 } & Experimental & Theoretical & Experimental & Theoretical \\
\hline BGA & $62^{\circ}<\phi_{1}<82^{\circ}$, & $-68 \pm 14^{\circ}$, & $-77^{\circ}<\phi_{2}<-67^{\circ}$, & $-69 \pm 11^{\circ}$, \\
trisac- & $61^{\circ}<\varphi 1<74^{\circ}$ & $51 \pm 25^{\circ}$ & $-109^{\circ}<\varphi_{2}<-86^{\circ}$ & $-101 \pm 26^{\circ}$ \\
charide & & & & \\
BGA- $68^{\circ}, 77^{\circ}$ & $82 \pm 11^{\circ}$, & $-68^{\circ},-90^{\circ}$ & $-69 \pm 8^{\circ}$, \\
ScFv & $68 \pm 7^{\circ}$ & & $-113 \pm 10^{\circ}$ \\
complex & & &
\end{tabular}

${ }^{a}$ Glycosidic torsion angles for the GalNAca $(1,3) \mathrm{Gal}\left(\phi_{1}, \varphi_{1}\right)$.

${ }^{b}$ Torsion angles for Fuca $(1,2) \mathrm{Gal}\left(\phi_{2}, \varphi_{2}\right)$.

would be dependent on interactions with this residue. Second, in the case of the mutational approach, poses were rejected if the Ala-Trp mutation led to irreconcilable steric clashes with the antigen (Figure 2B). All the docked poses obtained from each of these approaches were then scored using a CHI scoring function. After applying these criteria, both docking approaches identified essentially equivalent antigen poses $(0.48 \AA \mathrm{RMSD}$ between ligand positions) (Figure 2A), in which the C-6 atom of the GalNAc forms a $\mathrm{CH} / \pi$ stacking interaction with the $\operatorname{Trp}^{\mathrm{H} 96}$. This complex was selected for further analysis by MD simulation.

\section{STRUCTURAL STABILITY OF THE IMMUNE COMPLEXES Blood group $A$}

The final docked model of the blood group antigen A bound to the antibody remained stable during the course of a $50 \mathrm{~ns}$ simulation based on the RMSD of the ring atoms of the ligand, which remained between 2 and $4 \AA$ over the course of the simulation (Figure 5). An analysis of the ring conformational preferences showed that all three residues in the trisaccharide remained in the ${ }^{4} \mathrm{C}_{1}$ chair conformations. The $\phi$ - and $\psi$-glycosidic torsion angles for the GalNAc $\alpha(1,3) \mathrm{Gal}\left(\phi_{1}, \psi_{1}\right)$ and Fuc $\alpha(1,2) \mathrm{Gal}\left(\phi_{2}\right.$,

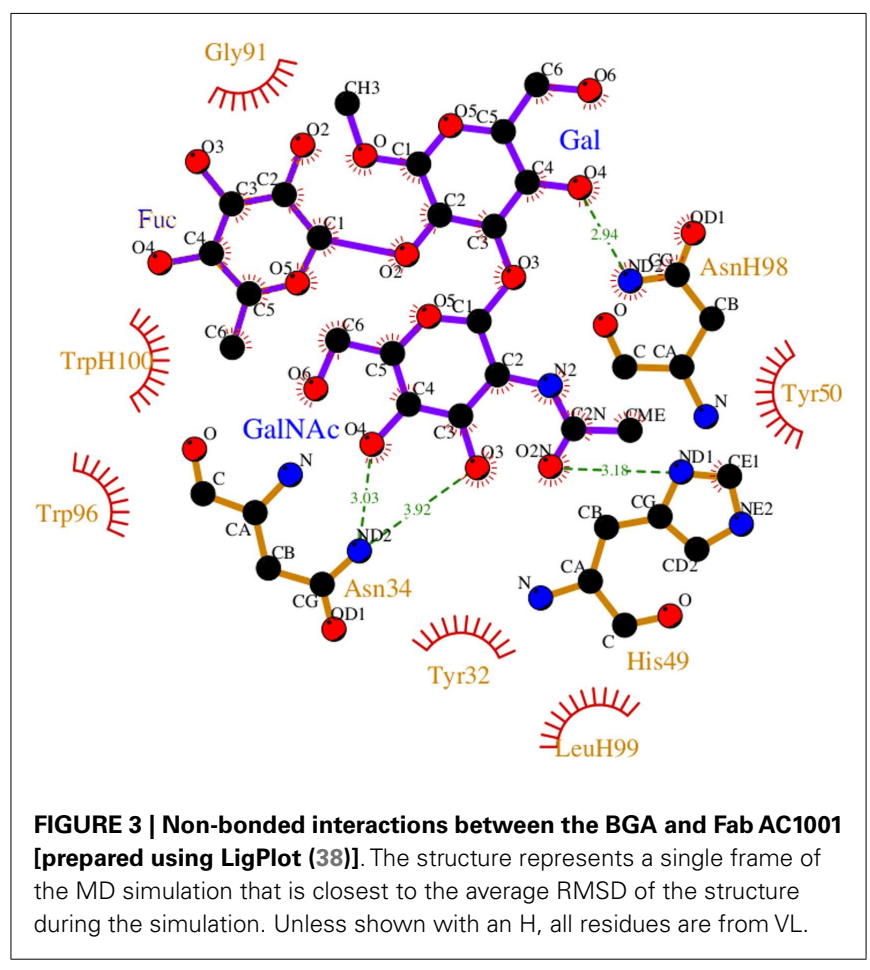

$\left.\Psi_{2}\right)$ linkages were monitored throughout both the simulations (BGA-scFv complex and BGA trisaccharide in solution) and the average values were found to be in agreement with the values observed for the same trisaccharide in the complex with Dolichos biflorus lectin as well as the conformations of the trisaccharide in solution (35) (Table 1). The stacking interactions between the GalNAc and $\operatorname{Trp}^{\mathrm{H} 96}$ interactions were characterized by the angle $(\theta)$ between the normals to the ring planes, and the distance $(R)$ between their centroids (36). For an ideal stacking conformation, $\theta$ should be around $180^{\circ}$ or $0^{\circ}$, and for $\mathrm{CH} / \pi$, it should be around $90^{\circ}$. The average $\theta$ value was close to the latter at $108^{\circ}$ (with a standard deviation of $9^{\circ}$ ) at a distance of $6.5 \AA$. 

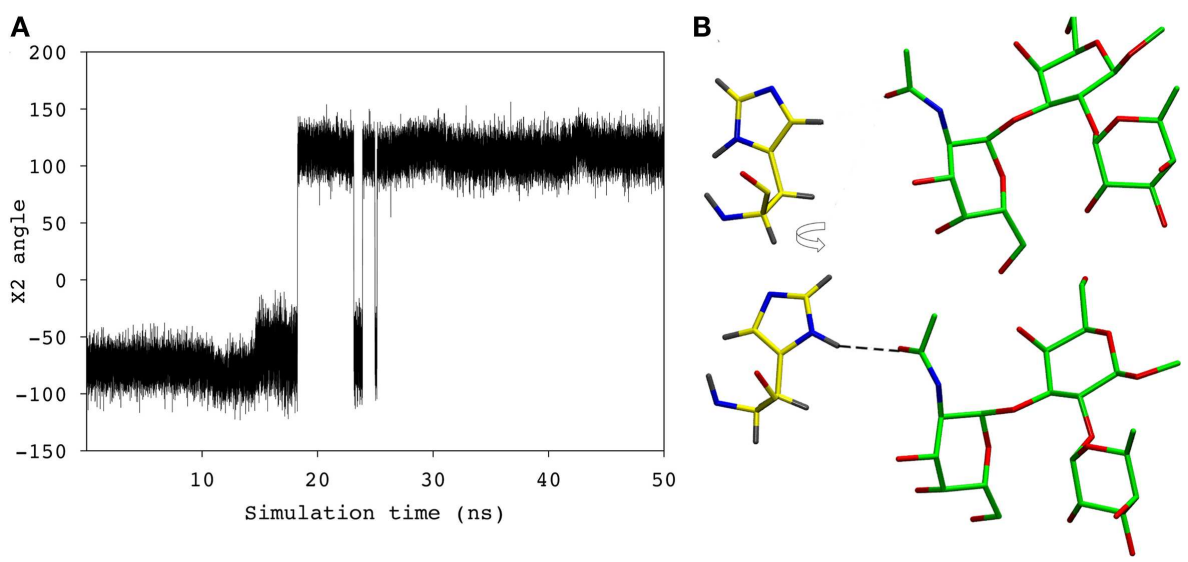

FIGURE 4 | (A) The $\chi_{2}$ angle of the His ${ }^{\llcorner 49}$ during the course of the simulation. (B) His ${ }^{\llcorner 49}$ (shown in yellow) during the first 18 ns of the simulation (top) and the remainder of the simulation (bottom).

Table 2 | Hydrogen bonds between BGA and the scFv during the MD simulation.

\begin{tabular}{|c|c|c|c|c|c|c|c|}
\hline \multicolumn{2}{|c|}{ Donor } & \multicolumn{2}{|c|}{ Acceptor } & \multicolumn{2}{|c|}{ MD period: 0-18 ns } & \multicolumn{2}{|c|}{ MD period: $18-50 \mathrm{~ns}$} \\
\hline & $\mathrm{O} 4$ & Asn ${ }^{\mathrm{L} 34}$ & $\mathrm{H} \delta 1$ & $3.1(0.22)$ & 32 & $3.0(0.17)$ & 77 \\
\hline & $\mathrm{O} 2 \mathrm{~N}$ & $\mathrm{His}^{\llcorner 49}$ & $\mathrm{H} \delta$ & $>3.5$ & - & $2.9(0.16)$ & 91 \\
\hline Gal & $\mathrm{O} 4$ & GalNAc & $\mathrm{H} 2 \mathrm{~N}$ & $3.2(0.17)$ & 65 & $3.2(0.17)$ & 31 \\
\hline
\end{tabular}

a Standard deviations in parentheses.

During the course of the MD simulation, the side chain of His ${ }^{\mathrm{L} 49}$ was observed to flip from its initial orientation $\left(\chi_{2}=\langle-73\rangle\right)$ to $(\langle 115\rangle)$ in which it could form a hydrogen bond with the $\mathrm{N}$ acetyl group of the GalNAc residue (Figures 3 and 4; Table 2). This interaction remained stable for the remainder of the $50 \mathrm{~ns}$ simulation. This side-chain flip may represent an example of induced fit during ligand binding, however, at the resolution of the present $\mathrm{X}$ ray data $(2.2 \AA)$, it is not possible to reliably discriminate between histidine $\chi_{2}$ rotamers $(37)$.

\section{Blood group $B$}

To probe the specificity of the antibody for antigen $\mathrm{B}$, the $\mathrm{scFv}$ was screened experimentally against an array of neoglycoconjugates including $\mathrm{ABO}$ and related blood group antigens. The screening confirmed the exclusive specificity of the antibody for BGA-related antigens (Tables S2 and S3 in Supplementary Material). Computational carbohydrate grafting (39) of the relevant glycans from the array onto the bound BGA trisaccharide in the scFv complex confirmed that all of the BGA- and BGB-related glycans could be accommodated in the binding pocket (Table S3 in Supplementary Material). Therefore, the lack of binding of the BGB-glycans does not appear to be due to steric collisions, but rather to the loss of affinity arising from the absence of the NAc group in the BGA congeners. MD simulation of the BGB-scFv complex was employed to examine the effect of the loss of the NAc moiety on the stability and affinity of the structural difference in the antigens on the stability and affinity of the putative immune complex. Despite the fact that the MD simulations of the two complexes (BGA and BGB) were started with the antigens aligned in identical binding modes, the $\mathrm{BGB}$ antigen dissociated from the antibody after a relatively short simulation period of $10 \mathrm{~ns}$. In order to eliminate the possibility that this instability arose due to artifacts from the conversion of the BGA to BGB antigen, two additional simulations were performed with independent initial atomic velocities. In both cases, the ligand appeared to dissociate from the antibody after approximately $10 \mathrm{~ns}$ (Figure 5). To enable comparison with the BGA complex, only the data from the initial stable $10 \mathrm{~ns}$ period of the BGB complex were chosen for analysis.

In antigen-scFv complexes, the Gal or GalNAc residues are flanked by residues $\mathrm{Tyr}^{\mathrm{L} 50}, \mathrm{Asn}^{\mathrm{L} 34}$ and $\mathrm{His}^{\mathrm{L} 49}$ on one side of the antigen (Group 1) and residues $\operatorname{Trp}^{\mathrm{H} 100}$ and $\operatorname{Trp}^{\mathrm{L} 96}$ (Group 2) on the other; the Fuc interacts with Gly ${ }^{\mathrm{L} 91}$ and $\mathrm{Asn}^{\mathrm{L} 92}$ (Group 3) (Figure 6). In contrast to the case of the BGA antigen, in the $\mathrm{BGB}-\mathrm{scFv}$ simulation $\mathrm{His}^{\mathrm{L} 49}$ does not form a stabilizing interaction with the terminal Gal residue. Additionally, the Gal and Fucl residues display enhanced flexibility owing to the loss of stabilizing interactions with residues from Groups 2 and 3. 


\section{INVOLVEMENT OF HIS ${ }^{\text {L99 }}$ IN BINDING AFFINITY}

All histidines in the $\mathrm{scFv}$ were protonated by default for modeling with a hydrogen atom at the $\delta$ nitrogen position. During the MD simulation of the BGA-scFv complex, the $\chi 2$ angle of His ${ }^{\mathrm{L} 49}$ flips $\left(-73^{\circ}\right.$ to $\left.115^{\circ}\right)$ enabling a hydrogen bond to form with the carbonyl moiety of the NAc group in the GalNAc residue in BGA, which would be expected to be significant for enhancing the stability of the BGA-scFv complex. In the BGB complex, the same $\mathrm{His}^{\mathrm{L} 49}$ forms an interaction with the non-terminal Gal residue. The interaction with $\mathrm{HisL}^{49}$ suggests that there might

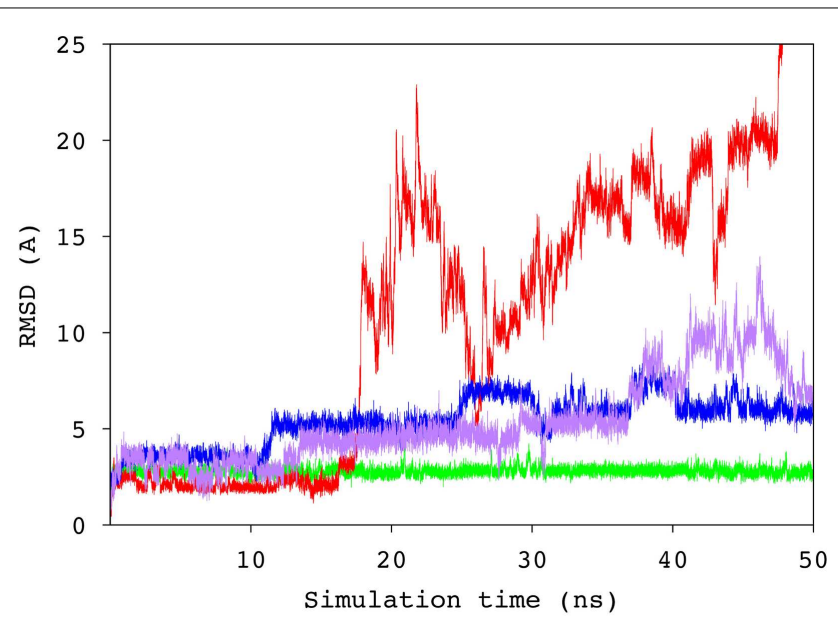

FIGURE 5 | Time series of the RMSD values for the ring atoms of the BGA (green) and BGB (from three independent simulations, blue, purple, and red) antigens, relative to the starting conformation of the complex. also be a $\mathrm{pH}$ dependence on binding; at lower $\mathrm{pH}$ all histidines would be positively charged, potentially enhancing the strength of the His ${ }^{\text {L9 }}$-BGA hydrogen bond, leading to higher binding affinity. This prediction was confirmed by BLI measurements, which showed a marked decrease in the apparent $K_{\mathrm{D}}$ as the $\mathrm{pH}$ dropped below the $\mathrm{p} K_{\mathrm{a}}$ of histidine (Figure 7 ). It should be noted that this protonation would not be localized to $\mathrm{His}^{\mathrm{L}}{ }^{49}$ nevertheless, no enhanced non-specific binding was observed at low $\mathrm{pH}$ for either

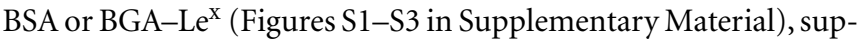
porting a role for a direct interaction between $\mathrm{His}^{\mathrm{L} 49}$ and the BGA antigen.

\section{BINDING ENERGY ANALYSIS}

A per-residue decomposition of the interaction energies in the immune complexes indicated that, in the case of BGA, the

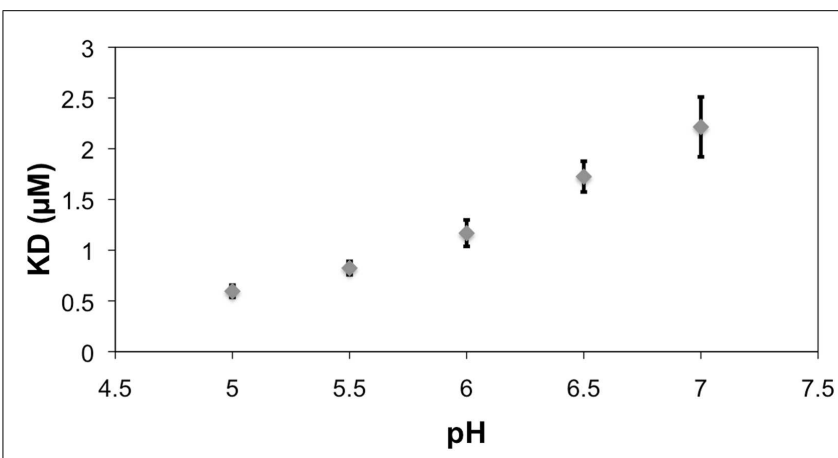

FIGURE 7 |The reference (BSA)-subtracted $\mathrm{pH}$ dependence of the apparent $K_{\mathrm{D}}$ for the interaction between scFv AC1001 and the BSA-BGA conjugate. Error bars are derived from replicates of five measurements. Note, the pKa of histidine is 6.04 (40).

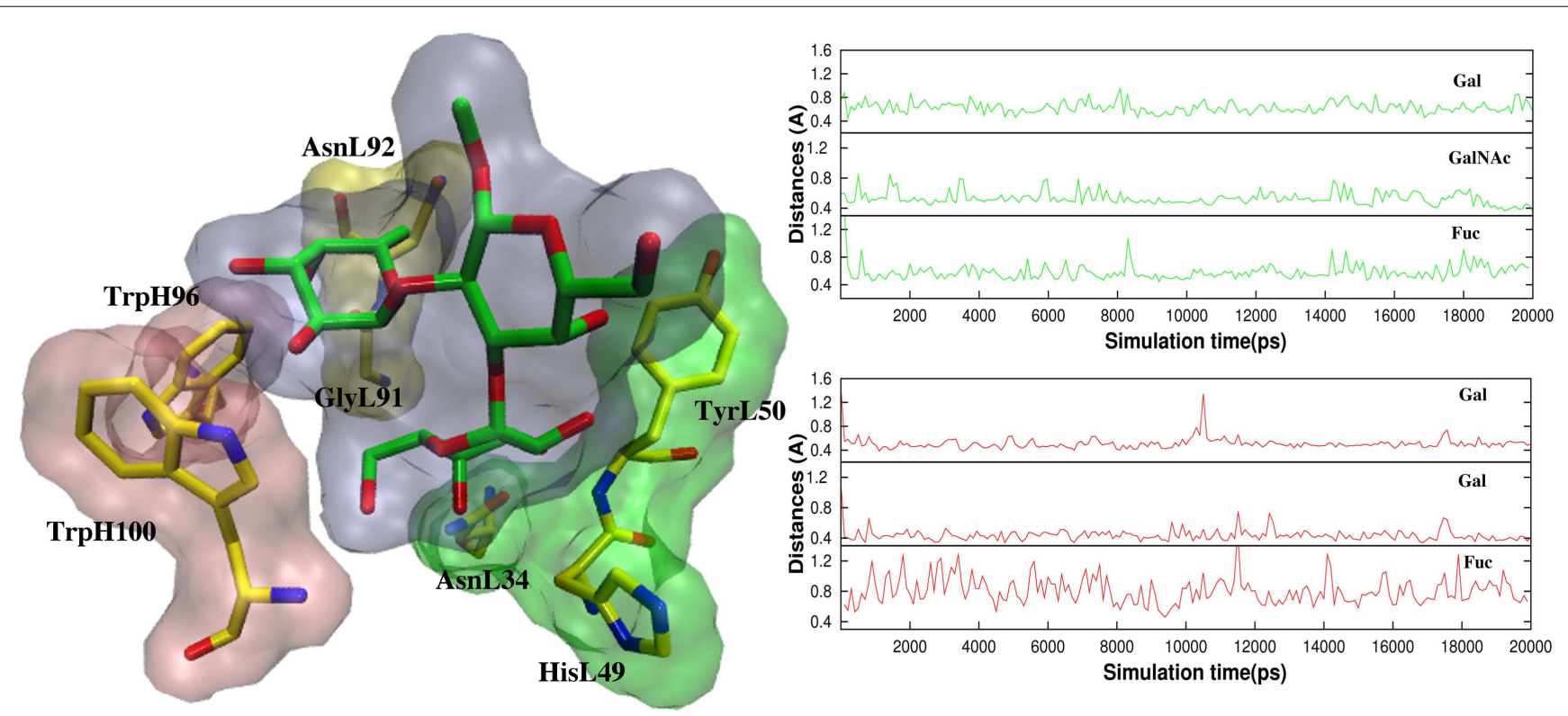

FIGURE 6 | (Left) The antigens are flanked by Tyr ${ }^{L 50}, \mathrm{Asn}^{\mathrm{L34}}$, and His ${ }^{\mathrm{L49}}$ (Group 1, green surface), and $\operatorname{Trp}^{\mathrm{H1} 100}$ and Trp ${ }^{\mathrm{L96}}$ (Group 2, pink surface). Fuc interacts with Gly $^{191}$ and Asn ${ }^{122}$ (Group3, yellow surface). (Right) Atomic fluctuations of residues Gal, GalNAc/Gal (BGA/BGB), and Fuc as a function of time. 
GalNAc residue contributed $25 \%(-8.1 \mathrm{kcal} / \mathrm{mol})$ toward the binding energy, compared to a reduced $(-4.3 \mathrm{kcal} / \mathrm{mol})$ contribution from the corresponding Gal residue in BGB (Table 3). This loss

Table 3 | Key ${ }^{\mathrm{a}}$ per-residue contributions toward the energy for the BGA and BGB-scFv complexes.

\begin{tabular}{|c|c|c|c|c|c|}
\hline Residue & vdW & Electrostatic & $\begin{array}{c}\text { Polar } \\
\text { desolvation }\end{array}$ & $\begin{array}{l}\text { Non-polar } \\
\text { desolvation }\end{array}$ & Total \\
\hline \multicolumn{6}{|l|}{ Antibody } \\
\hline \multirow[t]{2}{*}{ Tyr ${ }^{\llcorner 32}$} & $-4.2^{b}$ & -1.4 & 2.1 & -0.5 & -4.0 \\
\hline & $-3.0^{\mathrm{b}}$ & -1.7 & 2.0 & -0.5 & -3.2 \\
\hline \multirow[t]{2}{*}{ Gly ${ }^{L 91}$} & -0.8 & -4.8 & 3.2 & -0.2 & -2.5 \\
\hline & - & - & - & - & - \\
\hline \multirow[t]{2}{*}{$\operatorname{Trp}{ }^{H 100}$} & -2.5 & -1.2 & 1.7 & -0.3 & -2.2 \\
\hline & -2.8 & -1.0 & 1.2 & -0.4 & -2.9 \\
\hline \multirow[t]{2}{*}{$\operatorname{Trp} L 96$} & -1.4 & -1.1 & 0.7 & -0.2 & -2.0 \\
\hline & -0.7 & -0.3 & 0.2 & -0.1 & -0.9 \\
\hline \multirow[t]{2}{*}{ Asn $n^{\llcorner 34}$} & -0.8 & -1.6 & 0.9 & -0.1 & -1.6 \\
\hline & -0.6 & -3.7 & 1.5 & -0.1 & -2.9 \\
\hline \multirow[t]{2}{*}{ Asn ${ }^{H 98}$} & -2.2 & -2.9 & 4.0 & -0.5 & -1.5 \\
\hline & -2.8 & -1.6 & 3.8 & -0.5 & -1.1 \\
\hline \multirow[t]{2}{*}{ Asn ${ }^{L 92}$} & -1 & -2.9 & 2.6 & -0.2 & -1.5 \\
\hline & - & - & - & - & - \\
\hline \multirow[t]{2}{*}{ Tyr ${ }^{\llcorner 50}$} & -1.6 & -0.9 & 1.1 & -0.1 & -1.5 \\
\hline & -1.7 & -1.0 & 1.4 & -0.1 & -1.5 \\
\hline \multirow[t]{2}{*}{$\mathrm{Leu}^{\mathrm{H} 99}$} & -1.4 & -1 & 1 & -0.1 & -1.4 \\
\hline & -1.4 & -0.3 & 0.8 & -0.1 & -1 \\
\hline \multirow[t]{2}{*}{$\mathrm{His}^{\mathrm{L} 49}$} & -0.6 & -3.3 & 2.5 & -0.1 & -1.4 \\
\hline & -1.3 & -2.8 & 2.5 & -0.2 & -1.8 \\
\hline \multirow[t]{2}{*}{ Thr ${ }^{L 93}$} & -0.5 & -0.6 & 0.6 & 0.0 & -0.5 \\
\hline & - & - & - & - & - \\
\hline \multirow[t]{2}{*}{ Subtotal } & -17.1 & -20.5 & 20.5 & -2.2 & -19.3 \\
\hline & -11.3 & -12.4 & 13.4 & -2 & -15.3 \\
\hline \multicolumn{6}{|l|}{ Antigen } \\
\hline \multirow[t]{2}{*}{ Gal } & -3.1 & -0.4 & 1.2 & -0.4 & -2.7 \\
\hline & -6.2 & -3.7 & 5.2 & -0.9 & -5.6 \\
\hline \multirow[t]{2}{*}{ GalNAc/Gal } & -13.1 & -12.5 & 19.6 & -2.1 & -8.1 \\
\hline & -10.1 & -8.2 & 15.8 & -1.7 & -4.3 \\
\hline \multirow[t]{2}{*}{ Fuc } & -4.0 & -9.7 & 12.2 & -0.8 & -2.3 \\
\hline & -2.5 & -1.2 & 4.5 & -0.4 & 0.4 \\
\hline \multirow[t]{2}{*}{ Ligand total } & -20.2 & -22.6 & 32.9 & -3.2 & -13.2 \\
\hline & -18.9 & -13.2 & 25.4 & -1.4 & -9.6 \\
\hline
\end{tabular}

${ }^{a}$ Key residues defined as those that contribute $>0.5 \mathrm{kcal} / \mathrm{mol}$ to the total interaction energy for either the BGA or BGB in the complexes. Only the initial stable 10 ns period of the $B G B$ simulation was employed, whereas the entire 50 ns trajectory for BGA was analyzed.

b Upper row, values for BGA; lower, BGB. of $4 \mathrm{kcal} / \mathrm{mol}$ of interaction energy is the predominant difference between the two antigens, and would be enough to reduce the affinity by nearly 800 -fold, consistent with the lack of apparent binding of the BGB analogs in the glycan array screening. In addition, this analysis identified the residues that contributed significantly toward antigen binding.

In the BGA-scFv complex, residues from CDR L3 make the maximum contributions to binding $\left(\mathrm{Gly}^{\mathrm{L} 91}+\operatorname{Trp}^{\mathrm{L} 96}+\mathrm{Asn}^{\mathrm{L} 92}+\right.$ $\left.\mathrm{Thr}^{\mathrm{L} 93}=-7.2 \mathrm{kcal} / \mathrm{mol}\right)$ followed by $\mathrm{H3}\left(\mathrm{Asn}^{\mathrm{H} 98}+\mathrm{Trp}^{\mathrm{H} 100}+\right.$ $\left.\mathrm{Leu}^{\mathrm{H} 99}=-5.5 \mathrm{kcal} / \mathrm{mol}\right), \quad \mathrm{L} 1 \quad\left(\mathrm{Tyr}^{\mathrm{L} 32}+\mathrm{Asn}^{\mathrm{L} 34}=-4.5 \mathrm{kcal} / \mathrm{mol}\right)$, and $\mathrm{L} 2\left(\mathrm{Tyr}^{\mathrm{L} 50}=-1.02 \mathrm{kcal} / \mathrm{mol}\right)$. In contrast, in the case of BGB, the same residues from $\mathrm{L} 3$ contribute less than a total of $1 \mathrm{kcal} / \mathrm{mol}$ to the interaction energies. The most significant single residues are $\operatorname{Tyr}^{\mathrm{L} 32}, \mathrm{Gly}^{\mathrm{L} 91}, \operatorname{Trp}^{\mathrm{H} 100}$, and $\operatorname{Trp}^{\mathrm{L} 96}$, which each contributes more than $2 \mathrm{kcal} / \mathrm{mol}$ and together account for $50 \%$ of the total affinity. Residues Gly ${ }^{\mathrm{L} 91}$ and $\mathrm{Asn}^{\mathrm{L} 92}$ that form hydrogen bonds with the Fuc residue together contribute $-4.0 \mathrm{kcal} / \mathrm{mol}$ to the binding of BGA, but fail to make any stable interactions in the BGB simulation and therefore contribute negligibly to the affinity. It is these interactions that provide the predominant contributions to the preferential binding of the BGA antigen. While in the BGB complex, His ${ }^{\mathrm{L} 49}$ does not form any stable hydrogen bonds with the terminal Gal, it is able to form new, albeit transient, interactions with the non-terminal Gal for $30 \%$ of the stable simulation period. Therefore, while the per-residue contribution values indicate that $\mathrm{His}^{\mathrm{L} 49}$ makes a contribution greater than $-1.5 \mathrm{kcal} / \mathrm{mol}$ in both cases, the interactions it forms in BGA are more stable when compared to the interactions in BGB.

\section{CONCLUSION}

In this study, 3D models of the BGA and $\mathrm{BGB}$ trisaccharides in complex with scFv AC1001 were generated that provided a detailed atomic level rationalization of the interactions and dynamics responsible for antigen specificity. Quantification of the binding affinities identified key residues in the binding site that are predicted to contribute to specific and non-specific interactions with each antigen and led to the confirmed prediction of enhanced binding at lower $\mathrm{pH}$. The spontaneous dissociation of antigen $\mathrm{B}$ from the scFv-BGB complexes (in three different simulations) indicated that MD simulations confirm the known preference of this antibody for the A antigen, and support a role for MD simulations in overcoming limitations associated with ligand docking. The present study illustrates that integration of multiple experimental (affinity measurements, glycan array screening, and crystallography) and theoretical (ligand docking, MD simulation, and energy decomposition) methods provides a powerful platform for predicting the origin of antibody-carbohydrate specificity.

\section{AUTHOR CONTRIBUTIONS}

Spandana Makeneni, N. Martin Young, and Robert J. Woods conceived and designed the experiments. Spandana Makeneni, Ye Ji, and David C. Watson performed the experiments. Spandana Makeneni, Ye Ji, and Robert J. Woods analyzed the data. Spandana Makeneni, Ye Ji, N. Martin Young, David C. Watson, and Robert J. Woods contributed reagents/materials/analysis tools. Spandana Makeneni, N. Martin Young, and Robert J. Woods wrote the paper. 


\section{ACKNOWLEDGMENTS}

We would like to thank Dr. Jeffery C. Gildersleeve and W. Shea Wright for screening the scFv against the carbohydrate array. We wish to acknowledge the Consortium for Functional Glycomics grant number GM62116 for screening the antibody against their array. We thank the National Institutes for Health [R01 GM094919 (EUREKA) and P41 GM103390], as well as the Science Foundation of Ireland (08/IN.1/B2070) and the European Research Development Fund for support.

\section{SUPPLEMENTARY MATERIAL}

Included in the supplemental information are details and raw data from the BLI experiments. Results from screening of the $\mathrm{scFv}$ against both CFG and carbohydrate arrays (Dr. Jeffery Gildersleeve's group) are also presented in the supplemental material. The Supplementary Material for this article can be found online at http://www.frontiersin.org/Journal/10.3389/fimmu.2014.00397

\section{REFERENCES}

1. Landsteiner K. Zur Kenntnis der antifermentativen, lytischen und agglutinierenden Wirkungen des Blutserums und der Lymphe. Zentralbl Bakteriol (1900) 27(10):357-62.

2. Yamamoto F. Review: $A B O$ blood group system - ABH oligosaccharide antigens, anti-A and anti-B, A and B glycosyltransferases, and $\mathrm{ABO}$ genes. Immunohematology (2004) 20(1):3-22.

3. Chester MA, Olsson ML. The ABO blood group gene: a locus of considerable genetic diversity. Transfus Med Rev (2001) 15(3):177-200. doi:10.1053/tmrv. 2001.24591

4. Marionneau S, Cailleau-Thomas A, Rocher J, Le Moullac-Vaidye B, Ruvoën N, Clément $\mathrm{M}$, et al. $\mathrm{ABH}$ and Lewis histo-blood group antigens, a model for the meaning of oligosaccharide diversity in the face of a changing world. Biochimie (2001) 83(7):565-73. doi:10.1016/S0300-9084(01)01321-9

5. Williamson LM, Lowe S, Love EM, Cohen H, Soldan K, McClelland DB, et al. Serious hazards of transfusion (SHOT) initiative: analysis of the first two annual reports. BMJ (1999) 319(7201):16-9. doi:10.1136/bmj.319.7201.16

6. Sazama K. Reports of 355 transfusion-associated deaths: 1976 through 1985. Transfusion (1990) 30(7):583-90. doi:10.1046/j.1537-2995.1990.30790385515.x

7. Ozolek JA, Watchko JF, Mimouni F. Prevalence and lack of clinical significance of blood group incompatibility in mothers with blood type A or B. J Pediatr (1994) 125(1):87-91. doi:10.1016/S0022-3476(94)70131-8

8. Le Pendu J, Marionneau S, Cailleau-Thomas A, Rocher J, Le Moullac-Vaidye $\mathrm{B}$, Clement M. ABH and Lewis histo-blood group antigens in cancer. APMIS (2001) 109(1):9-31. doi:10.1111/j.1600-0463.2001.tb00011.x

9. Dabelsteen E, Gao S. ABO blood-group antigens in oral cancer. J Dent Res (2005) 84(1):21-8. doi:10.1177/154405910508400103

10. Anstee DJ. The relationship between blood groups and disease. Blood (2010) 115(23):4635-43. doi:10.1182/blood-2010-01-261859

11. Ceravolo IP, Sanchez BAM, Sousa TN, Guerra BM, Soares IS, Braga EM, et al. Naturally acquired inhibitory antibodies to Plasmodium vivax Duffy binding protein are short-lived and allele-specific following a single malaria infection. Clin Exp Immunol (2009) 156(3):502-10. doi:10.1111/j.1365-2249. 2009.03931.x

12. DeMarco ML, Woods RJ. Structural glycobiology: a game of snakes and ladders. Glycobiology (2008) 18(6):426-40. doi:10.1093/glycob/cwn026

13. Woods R, Yongye A. Computational techniques applied to defining carbohydrate antigenicity. In: Kosma P, Müller-Loennies S, editors. Anticarbohydrate Antibodies. Vienna: Springer (2012). p. 361-83.

14. Paula S, Monson N, Ball WJ Jr. Molecular modeling of cardiac glycoside binding by the human sequence monoclonal antibody 1B3. Proteins (2005) 60(3):382-91. doi:10.1002/prot.20484

15. Woods RJ, Tessier MB. Computational glycoscience: characterizing the spatial and temporal properties of glycans and glycan-protein complexes. Curr Opin Struct Biol (2010) 20(5):575-83. doi:10.1016/j.sbi.2010.07.005

16. Nivedha AK, Makeneni S, Foley BL, Tessier MB, Woods RJ. Importance of ligand conformational energies in carbohydrate docking: sorting the wheat from the chaff. J Comput Chem (2014) 35(7):526-39. doi:10.1002/jcc.23517
17. Thomas R, Patenaude SI, MacKenzie CR, To R, Hirama T, Young NM, et al. Structure of an anti-blood group A Fv and improvement of its binding affinity without loss of specificity. J Biol Chem (2002) 277(3):2059-64. doi:10.1074/jbc. M104364200

18. Trott O, Olson AJ. AutoDock Vina: improving the speed and accuracy of docking with a new scoring function, efficient optimization, and multithreading. J Comput Chem (2010) 31(2):455-61. doi:10.1002/jcc.21334

19. Hadden JA, Tessier MB, Fadda E, Woods RJ. Calculating binding free energies for protein-carbohydrate complexes. Methods in Molecular Biology: Glycoinformatics (in press).

20. Morris GM, Huey R, Lindstrom W, Sanner MF, Belew RK, Goodsell DS, et al. AutoDock4 and AutoDockTools4: automated docking with selective receptor flexibility. J Comput Chem (2009) 30(16):2785-91. doi:10.1002/jcc.21256

21. Gasteiger J, Marsili M. Iterative partial equalization of orbital electronegativity - a rapid access to atomic charges. Tetrahedron (1980) 36(22):3219-28. doi:10.1016/0040-4020(80)80168-2

22. Case DA, Darden TA, Cheatham TE, Simmerling CL, Wang J, Duke RE, et al. AMBER 12. San Francisco, CA: University of California (2012).

23. Götz AW, Williamson MJ, Xu D, Poole D, Le Grand S, Walker RC. Routine microsecond molecular dynamics simulations with AMBER on GPUs. 1. Generalized born. JChem Theory Comput (2012) 8(5):1542-55. doi:10.1021/ct200909j

24. Hornak V, Abel R, Okur A, Strockbine B, Roitberg A, Simmerling C. Comparison of multiple Amber force fields and development of improved protein backbone parameters. Proteins (2006) 65(3):712-25. doi:10.1002/prot.21123

25. Kirschner KN, Yongye AB, Tschampel SM, González-Outeiriño J, Daniels CR, Foley BL, et al. GLYCAM06: a generalizable biomolecular force field. Carbohydrates. J Comput Chem (2008) 29(4):622-55. doi:10.1002/jcc.20820

26. Jorgensen WL, Chandrasekhar J, Madura JD, Impey RW, Klein ML. Comparison of simple potential functions for simulating liquid water. J Chem Phys (1983) 79(2):926-35. doi:10.1063/1.445869

27. Ryckaert J-P, Ciccotti G, Berendsen HJC. Numerical integration of the cartesian equations of motion of a system with constraints: molecular dynamics of n-alkanes. J Comput Phys (1977) 23(3):327-41. doi:10.1016/0021-9991(77) 90098-5

28. Darden T, York D, Pedersen L. Particle mesh Ewald: an $N \cdot \log (\mathrm{N})$ method for Ewald sums in large systems. J Chem Phys (1993) 98(12):10089-92. doi:10.1063/1.464397

29. Roe DR, Cheatham TE. PTRAJ and CPPTRAJ: software for processing and analysis of molecular dynamics trajectory data. J Chem Theory Comput (2013) 9(7):3084-95. doi:10.1021/ct400341p

30. Kollman PA, Massova I, Reyes C, Kuhn B, Huo S, Chong L, et al. Calculating structures and free energies of complex molecules: combining molecular mechanics and continuum models. Acc Chem Res (2000) 33(12):889-97. doi:10.1021/ar000033j

31. Srinivasan J, Miller J, Kollman PA, Case DA. Continuum solvent studies of the stability of RNA hairpin loops and helices. J Biomol Struct Dyn (1998) 16(3):671-82. doi:10.1080/07391102.1998.10508279

32. Onufriev A, Bashford D, Case DA. Exploring protein native states and largescale conformational changes with a modified generalized born model. Proteins (2004) 55(2):383-94. doi:10.1002/prot.20033

33. Proctor Elizabeth A, Yin S, Tropsha A, Dokholyan Nikolay V. Discrete molecular dynamics distinguishes nativelike binding poses from decoys in difficult targets. Biophys J (2012) 102(1):144-51. doi:10.1016/j.bpj.2011.11.4008

34. Cygler M, Rose DR, Bundle DR. Recognition of a cell-surface oligosaccharide of pathogenic Salmonella by an antibody Fab fragment. Science (1991) 253(5018):442-5. doi:10.2307/2878892

35. Casset F, Peters T, Etzler M, Korchagina E, Nifant'ev N, Pérez S, et al. Conformational analysis of blood group A trisaccharide in solution and in the binding site of Dolichos biflorus lectin using transient and transferred nuclear Overhauser enhancement (NOE) and rotating-frame NOE experiments. Eur J Biochem (1996) 239(3):710-9. doi:10.1111/j.1432-1033.1996.0710u.x

36. Ford MG, Weimar T, Kohli T, Woods RJ. Molecular dynamics simulations of galectin-1-oligosaccharide complexes reveal the molecular basis for ligand diversity. Proteins (2003) 53(2):229-40. doi:10.1002/prot.10428

37. Glusker JPL, Mitchell L, Rossi M. Crystal structure analysis for chemists and biologists. J Chem Educ (1995) 72(3):A73. doi:10.1021/ed072pA73.9

38. Wallace AC, Laskowski RA, Thornton JM. LIGPLOT: a program to generate schematic diagrams of protein-ligand interactions. Protein Eng (1995) 8(2):127-34. doi:10.1093/protein/8.2.127 
39. Tessier MB, Grant OC, Heimburg-Molinaro J, Smith D, Jadey S, Gulick AM, et al. Computational screening of the human TF-glycome provides a structural definition for the specificity of anti-tumor antibody JAA-F11. PLoS One (2013) 8(1):e54874. doi:10.1371/journal.pone.0054874

40. Wood EJ. Data for biochemical research (third edition) by R M C Dawson, D C Elliott, W H Elliott and K M Jones. Biochem Educ (1987) 15(2):97. doi:10.1016/0307-4412(87)90110-5

Conflict of Interest Statement: The authors declare that the research was conducted in the absence of any commercial or financial relationships that could be construed as a potential conflict of interest.
Received: 02 July 2014; accepted: 05 August 2014; published online: 22 August 2014. Citation: Makeneni S, Ji Y, Watson DC, Young NM and Woods RJ (2014) Predicting the origins of anti-blood group antibody specificity: a case study of the ABOA-and B-antigens. Front. Immunol. 5:397. doi: 10.3389/fimmu.2014.00397

This article was submitted to Immunotherapies and Vaccines, a section of the journal Frontiers in Immunology.

Copyright (c) 2014 Makeneni, Ji, Watson, Young and Woods. This is an open-access article distributed under the terms of the Creative Commons Attribution License (CC $B Y)$. The use, distribution or reproduction in other forums is permitted, provided the original author(s) or licensor are credited and that the original publication in this journal is cited, in accordance with accepted academic practice. No use, distribution or reproduction is permitted which does not comply with these terms. 